\section{Análise comparativa de embalagens secundárias e bulas de medicamentos contendo Panax ginseng C. A. Meyer}

\author{
A comparative analysis of inner wrapping and \\ package inserts for medicines containing \\ Panax ginseng C. A. Meyer
}

1 Serviço de Medicamentos, Instituto Adolfo Lutz,

São Paulo, Brasil.

2 Faculdade de Ciências Farmacêuticas, Universidade de São Paulo, São Paulo, Brasil.

Correspondência M. T. Auricchio Serviço de Medicamentos, Instituto Adolfo Lutz. Av. Dr. Arnaldo 355, São Paulo, SP 01246-902, Brasil. mauricch@ial.sp.gov.br

\section{Abstract}

The information provided on package inserts and inner wrapping of eight products containing Panax ginseng from different manufacturers was compared internally and checked against data from the scientific literature. The inserts included extensive text, containing abundant information on indications for use, but no scientific evidence in humans. All the inserts lacked information on potential adverse effects and drug interaction. There was no standardization as to dose regimens, particularly in relation to the dried extract and ginsenoside concentration. The eight inserts thus showed no concern over standardization, indication for usage, or possible side effects and drug interactions.

Panax ginseng; Medicine Package; Drug Utilization
Mariangela Tirico Auricchio 1

Mônica Arcon Batistic-Longatto 1

Maria Aparecida Nicoletti 2

\section{Introdução}

A raiz de Panax ginseng C. A. Meyer, de cor amarelo-acinzentado, tem aspecto fusiforme ou cilíndrico e a forma retorcida, com raízes secundárias que, após secagem e pulverização, originam pó amarelo-claro com odor característico, levemente adocicado e amargo ao final; exibe abundantes cristais de oxalato de cálcio, em formato de cachos, e grãos de amido característicos quando observados ao microscópio. É encontrada desde as montanhas da China (Manchúria), Coréia, Japão até o leste da Sibéria, Rússia. Seus principais constituintes químicos são as saponinas triterpênicas, conforme assinalam Washida \& Kitanaka 1, Park et al. 2, sendo o pó e o extrato seco das raízes de ginseng comercializados, preferencialmente, na forma de cápsulas ou comprimidos.

Segundo dados da indústria farmacêutica, no Brasil, os fitoterápicos movimentam $400 \mathrm{mi}$ lhões de Dólares por ano, representando $7 \%$ do segmento farmacêutico, com perspectiva de aumento nos próximos anos ${ }^{3}$. O seu consumo é prática comum, também, em outros países, como os Estados Unidos 4 , onde mais de um terço da população faz uso de alguma forma de medicina não convencional além de suplementos dietéticos ou, então, de dietas com megavitaminas para a cura das doenças 5 .

No Brasil, muitos medicamentos fitoterápicos são classificados como medicamentos de venda livre, ou seja, sua utilização ocorre, normalmen- 
te, sem a intervenção do prescritor, tornando-se imperativo, portanto, que as informações fornecidas ao paciente sejam confiáveis, corretas e de acordo com a literatura científica.

Por se tratar de droga de origem vegetal muito conhecida e utilizada, pesquisadores têm se dedicado à investigação das propriedades do ginseng, a fim de comprovar os efeitos alegados no uso popular dessa droga. Os estudos sobre a atividade farmacológica do $P$. ginseng são numerosos e abrangentes 6,7,8. Choi et al. 9, Jeong et al. 10, Van Kampen et al. 11, Park et al. 12, Liou et al. 13 e Joo et al. 14 vêm se aprofundando até o momento na elucidação de suas propriedades terapêuticas, mas a Organização Mundial da Saúde (OMS) 15 já reconhece a atividade profilática e restauradora do ginseng como comprovada cientificamente.

Singh et al. 16 apresentaram evidências demonstrando que os ginsenosídeos ampliam o fornecimento de energia durante o exercício prolongado, por aumentarem a capacidade do músculo esquelético em oxidar ácidos graxos. Kumar et al. 17 constataram que o ginseng inibe a lipoperoxidação, impedindo, assim, a formação de radicais livres.

Nessa mesma linha, Yokozawa et al. 18 estudaram o relacionamento entre envelhecimento e defesa do sistema antioxidativo e, avaliando os efeitos dos ginsenosídeos Rd, concluíram que o ginseng atenuou os danos oxidativos responsáveis pela intervenção no estado redox do sistema glutationa/glutationa dissulfeto.

Estudo com o ginsenosídeo Rgl foi desenvolvido por Shen \& Zhang 19, sugerindo que essa substância esteja envolvida na regulação da proliferação de células precursoras do hipocampo, sendo este um mecanismo antienvelhecimento exercido pelo ginseng.

Com estudos em animais, Kim \& Park 20 observaram que o extrato de $P$. ginseng administrado intraperitonealmente em camundongos resultou na diminuição do bloqueio da ACTH na glândula adrenal, induzidos pelo estresse, levando ao aumento da concentração plasmática de corticosterona.

Inúmeros outros efeitos, como ações antiinflamatória, antioxidante e anticancerígena, além de melhoria das funções psicológicas, imunológicas e outras associadas ao diabetes, foram relatados por Kiefer \& Pantuso 21. Outros pesquisadores, Radad et al. 22 e Mizumaki et al. 23, por exemplo, sugeriram em seus achados que diferentes frações de extrato de ginseng possuem ações neurotróficas.

As atividades hepatoprotetora e regeneradora das funções hepáticas têm sido assinaladas in vitro, por Kim et al. 24 , pela sua atuação na pro- teção dos microssomos hepáticos de ratos em face do tetracloreto de carbono. Hikino 25, Kwon et al. ${ }^{26}$ e Kwon \& Jang 27 assinalaram in vivo essa atividade após a administração de ginseng por via oral em cães e ratos.

Gross et al. ${ }^{28}$, estudando os efeitos do extrato de ginseng padronizado (G115) sobre as funções pulmonares, assinalaram que houve melhora nos parâmetros medidos em pacientes com doença pulmonar obstrutiva crônica moderadamente severa.

Park et al. 2,12 observaram efeito estabilizador sobre a membrana celular, in vitro e in vivo, exercido pelos ginsenosídeos, o que explicaria a ação antiinflamatória decorrente de processos alérgicos. Choo et al. 29 assinalaram a presença do composto K, o qual seria o principal metabólito obtido dos ginsenosídeos pela ação das bactérias intestinais e que apresenta propriedades antialérgicas.

Outros estudos abordam o aspecto imunológico, como o desenvolvido por Joo et al. 14, que, empregando o ginsenosídeo Rg3, obtiveram melhora na proliferação de células de baço e medula óssea de camundongos. O extrato de ginseng, quando administrado intraperitonialmente em camundongos, foi capaz de regular a produção de anticorpos, aumentando a produção de citocinas, aspecto assinalado por Liou et al. 13 .

Vários autores estudaram os efeitos hipolipidêmicos do ginseng, entre eles Seung-Hwan \& Kyung-Shin ${ }^{30}$, Yoon et al. ${ }^{31}$ e Kim et al. ${ }^{32}$, que alegaram que os resultados se devem ao potencial antioxidante do ginseng.

Oshima et al. 33, Konno et al. 34,35 e Tomoda et al. 36 isolaram componentes do P. ginseng com atividade hipoglicemiante, que, à época, denominaram panaxanas. Dey et al. 37 compararam os efeitos da raiz e do fruto de $P$. ginseng, constatando que o segundo exerce ação normoglicêmica posprandial mais acentuada em camundongos com profunda obesidade (modelo semelhante a humanos com diabetes tipo II) do que as raízes. Sotaniemi et al. ${ }^{38}$ preconizaram o uso de ginseng em pacientes com diabetes não insulino-dependentes.

Outros autores demonstraram ações do ginseng no trato gastrintestinal. Min et al. 39 mostraram que alguns ginsenosídeos específicos da fração panaxitriol exibem ação antagônica a receptores relacionados a náuseas e vômitos; já Hashimoto et al. 40 assinalaram que os ginsenosídeos $\mathrm{Rbl}(4)$ e $\mathrm{Rd}(7)$ aceleram os movimentos do intestino em camundongos e Jeong et al. 10 indicaram que o efeito antiulceroso se dá pelo aumento na produção de muco.

Apesar de numerosos estudos que investigam a ação do ginseng como anticancerígeno, não há 
evidências conclusivas sobre esta ação. Por um lado, Chang et al. 41 afirmaram que ainda não há experimentos que comprovem clinicamente a atividade do ginseng em câncer; por outro, Helms 42 avaliou que o $P$. ginseng alivia o câncer por meio de ação antiinflamatória, antioxidante e mecanismos apoptóticos. Em estudo realizado, Oh et al. 43 relataram que os metabólitos do ginsenosídeo 20-(S)-protopanaxatriol, gerado pela ação das bactérias intestinais, podem ser agentes preventivos do câncer e de outras doenças inflamatórias.

Entre os adeptos da fitoterapia, é muitas vezes comum o pensamento de que as plantas medicinais sejam seguras e eficazes, sem os efeitos colaterais comuns aos medicamentos sintéticos Contudo, a planta medicinal também é um xenobiótico, que passará por biotransformação ao ser introduzida no organismo e, como tal, potencialmente tóxica, até prova em contrário, segundo Lapa et al. ${ }^{44}$.

Mesmo sendo uma das drogas mais populares do mundo e o segundo fitoterápico mais vendido nos Estados Unidos em 2000, com vários estudos clínicos realizados para se verificar sua eficácia, informações oriundas destes estudos em relação a possíveis efeitos adversos do ginseng são escassas e muitas vezes difíceis de serem analisadas 45 . Punnonen \& Lukola 46 e Koren 47 já consideravam a hipótese de que os ginsenosídeos ligam-se aos receptores hormonais, uma vez que vários sintomas do uso do ginseng são parecidos com os causados pelos hormônios estrogênicos, como, por exemplo, a presença de nódulos nas mamas em mulheres no período pós-menopausal, conforme Awang 48 .

Mais recentemente, Lee et al. 49 verificaram que o ginsenosídeo Rh1 liga-se a receptores dos hormônios esteroidais, para estrógenos, atuando como um fito-hormônio fraco.

Coon \& Ernst 45 fizeram revisão sobre eventos adversos e interações medicamentosas relacionadas ao uso de $P$. ginseng e relataram que os dados fornecidos pelas agências mundiais de saúde para eventos adversos provocados pelo ginseng são de casos raros e isolados, havendo pouca informação disponível dos próprios fabricantes. Já a OMS 15, até maio de 2001, relatava, em 18 países, 378 eventos adversos relacionados ao uso do ginseng isolado ou em associação com outras substâncias. A OMS 15, Dega et al. 50, Coon \& Ernst 45, Vasquez \& Aguera-Ortiz 51 e Tsung \& Cheng 52 citam as seguintes reações adversas mais comumente encontradas para o uso do ginseng: dor de cabeça, desordens do sono e gastrintestinais, manifestações alérgicas, hipertensão arterial, nervosismo, irritabilidade, hiperestrogenismo, síndrome de Stevens-Johnson e ação hipoglicemiante. Recentemente, Wiwanitkit \& Taungjaruwinai 53 também referiram um caso de reação alérgica sistêmica após o uso de ginseng.

Em relação às interações medicamentosas, Kiefer \& Pantuso 21 já relataram que o ginseng, quando ingerido, era bem tolerado, embora, pudesse interagir com outros fármacos. Janetzky \& Morreale 54 , Kuo et al. 55 e Miller 56 citaram algumas interações observadas no uso de ginseng concomitantemente a outras drogas, como a varfarina, um potente agente anticoagulante, apontado também por Dasgupta 57 , Sparreboom et al. 58, Yuang et al. 59 e Myers 60 .

Jones \& Runikis 61, Dasgupta 57 e Sparreboom et al. 58 reportaram interações com a fenelzina (inibidor da MAO) e com a nifedipina (bloqueador dos canais de cálcio). Outra abordagem foi estudada por Merino et al. 62 , que concluíram haver possibilidade de interação do ginseng com os benzimidazóis (antiparasitários).

A ingestão de álcool com o ginseng provoca um decréscimo da concentração plasmática de álcool em 32,5\%, segundo Izzo \& Ernst 63 e Sparreboom 58 .

No Brasil, com a intensificação da farmacovigilância, é esperada melhor conscientização sobre a ocorrência de eventos adversos relacionados ao uso de fitoterápicos, além da divulgação do conhecimento de possíveis intercorrências resultantes de interações medicamentosas. Essa ação, certamente, trará uma valiosa contribuição para a alteração da crença equivocada e culturalmente estabelecida a respeito da "segurança" desse tipo de medicamento.

Sendo assim, com o objetivo de verificar as informações disponíveis ao consumidor nas bulas dos medicamentos, foi feito estudo no qual se comparam estas informações com as da literatura e com a legislação vigente.

Os principais aspectos abordados para a realização da análise das informações disponibilizadas foram indicações de uso, eventos adversos e interações medicamentosas para fitoterápicos contendo ginseng, ou em medicamentos que apresentam ginseng em associação com outras substâncias, como vitaminas e sais minerais.

\section{Material e métodos}

As embalagens e bulas de oito amostras de preparações contendo $P$. ginseng, de sete diferentes fabricantes, encontradas no mercado da cidade de São Paulo, Brasil, foram analisadas, ressaltando-se que esse total era o número de especialidades disponíveis na época para comercialização. Destas, quatro eram associações de extrato seco 
de raiz de $P$. ginseng com vitaminas e sais minerais e quatro eram pós de extratos secos de raiz de P. ginseng. Todas as preparações apresentavamse como cápsulas ou comprimidos revestidos e sete continham bula.

O estudo, realizado com base na legislação vigente-Lei $n^{o}$. 6.360/76 64, Decreto $n^{o} .79 .094 / 7765$ e as resoluções da ANVISA ( $R D C n^{\circ} .92 / 0066, R D C$ $n^{o} .47 / 01$ 67, RDC no. 140/03 68, RDC no. 333/0369 e $\left.R D C n^{\circ} .8 / 0470\right)$-, consistiu na comparação entre as informações apresentadas nas embalagens e bulas das amostras, relativas às indicações de uso e posologia, reações adversas e interações medicamentosas, com aquelas descritas na literatura científica consultada até o final do ano de 2004, disponíveis em base de dados MEDLINE e $M E D S C A P E$. A busca foi realizada por meio das palavras-chave: "Panax ginseng", "side effects", "drug and food interactions", "pharmacological and toxicological properties".

\section{Resultados}

Como observamos neste trabalho, não há homogeneidade nas informações ao paciente sobre indicações de uso (Tabela 1), efeitos adversos (Tabela 2) e interações medicamentosas (Tabela 3) de produtos contendo P. ginseng, entre as oito amostras estudadas. A Tabela 4 evidencia a falta de informação de interações medicamentosas das amostras comparativamente às disponíveis na literatura científica e a Tabela 5 caracteriza a diversidade da posologia encontrada nos produtos analisados.

\section{Discussão}

O rótulo e a bula são os instrumentos de comunicação utilizados pelo fabricante para informar sobre seu produto ao usuário de medicamento e devem assegurar informações claras e precisas, a fim de evitar que ocorrências inesperadas ou indesejadas afetem a segurança e a eficácia do produto.

De acordo com a legislação vigente para rotulagem, os medicamentos fitoterápicos devem conter, além do nome comercial, a nomenclatura botânica e o termo "Fitoterápico" bem discriminado; não deve estar expresso que se trata de medicamento "natural", para que não haja a idéia de produto inócuo ou, então, que possua propriedades especiais. Nestes textos, a exigência para os medicamentos fitoterápicos limita-se a definir apenas os itens importantes que devem constar do relatório de petição de registro. Ainda que a legislação para fitoterápicos, a $R D C n^{o} .48 / 0470$, não defina regras específicas para rotulagem, as bulas e os rótulos devem obedecer ao estabelecido na $R D C n^{o} .333 / 0369$ e RDC $n^{o} .140 / 0368$.

Na Tabela 1, onde são listadas as indicações de uso descritas nas embalagens e bulas, observa-se concordância entre as bulas analisadas apenas quanto aos efeitos regeneradores do ginseng; somente na amostra 5 houve a preocupa-

Tabela 1

Indicações de uso de preparações industrializadas contendo Panax ginseng descritas na literatura científica comparadas às das embalagens e bulas de oito amostras comercializadas na cidade de São Paulo, Brasil, 2004-2005.

\begin{tabular}{|c|c|c|c|c|c|c|c|c|}
\hline \multirow[t]{2}{*}{ Indicações de uso descritas em literatura } & \multicolumn{8}{|c|}{ Especialidades farmacêuticas analisadas } \\
\hline & 1 * & 2 & 3 & 4 & 5 & 6 & 7 & 8 \\
\hline $\begin{array}{l}\text { Efeitos regeneradores (atividade profilática e restauradora do } \\
\text { desempenho físico e mental) } 6,7,8,9,10,11,12,13,14,15,16,17,18,19,20,22,23,28\end{array}$ & + & + & + & + & + & + & + & + \\
\hline Hepatoproteção 24,25,26,27 & - & - & - & - & + & - & - & - \\
\hline Disfunção erétil 75 & - & + & - & - & + & + & - & - \\
\hline Antiemético 12,39 & - & - & - & - & - & - & - & - \\
\hline Tratamento de desordens gastrintestinais (gastrite e úlcera) $10,39,40$ & - & - & - & - & + & - & - & - \\
\hline Atuação em metabolismo de lipídeos e glicemia $30,31,32,33,34,35,36,37,38$ & - & - & - & - & + & + & - & - \\
\hline Ação imunológica 2,12,13,14,21,29 & - & - & - & + & - & - & - & - \\
\hline Câncer 21,41,42,43 & - & - & - & - & - & - & - & - \\
\hline Efeito estrogênico $46,47,48,49$ & - & - & - & - & - & - & - & - \\
\hline
\end{tabular}

* Informação contida no cartucho somente.

+ Informação presente na bula.

- Informação ausente na bula. 
Reações adversas descritas na literatura científica comparadas às de embalagens e bulas de oito amostras de preparações industrializadas contendo Panax ginseng comercializadas na cidade de São Paulo, Brasil, 2004-2005.

\begin{tabular}{|c|c|c|c|c|c|c|c|c|}
\hline \multirow[t]{2}{*}{ Reações adversas descritas em literatura } & \multicolumn{8}{|c|}{ Especialidades farmacêuticas analisadas } \\
\hline & 1 & 2 & 3 & 4 & 5 & 6 & 7 & 8 \\
\hline Manifestações alérgicas (erupções cutâneas, urticária e prurido) 15,50,53 & - & - & + & + & + & + & + & - \\
\hline Insônia 15 & - & + & + & + & + & + & + & - \\
\hline Manifestações gastrintestinais (diarréia, náusea, vômito, dor no estômago) 15,45 & - & - & + & + & + & + & - & - \\
\hline Sangramento vaginal 15,56 & - & - & + & - & - & - & - & - \\
\hline Hipertensão arterial 15 & - & - & + & - & + & + & - & - \\
\hline Nervosismo/irritabilidade/palpitações 15, 51 & - & - & + & - & + & + & + & - \\
\hline Cefaléia 45 & - & - & - & - & - & - & + & - \\
\hline Hiperestrogenismo 15,56 & - & - & + & - & - & - & + & - \\
\hline Ação hipoglicemiante 52 & - & - & - & - & - & - & - & - \\
\hline
\end{tabular}

+ Informação presente na bula.

- Informação ausente na bula.

Interações medicamentosas descritas em embalagens e bulas de oito amostras de preparações industrializadas contendo Panax ginseng, comercializadas na cidade de São Paulo, Brasil, 2004-2005.

\begin{tabular}{|c|c|c|c|c|c|c|c|c|}
\hline \multirow[t]{2}{*}{ Interações descritas em bula } & \multicolumn{8}{|c|}{ Especialidades farmacêuticas analisadas } \\
\hline & 1 & 2 & 3 & 4 & 5 & 6 & 7 & 8 \\
\hline Nas doses recomendadas não apresenta significativas interações medicamentosas & * & - & - & - & - & + & - & - \\
\hline Até o momento não são descritas interações com alimentos ou medicamentos & * & + & + & + & - & - & - & + \\
\hline Drogas antidepressivas & * & - & - & - & - & - & + & - \\
\hline Heterosídeos digitálicos (etionamida, griseofulvina, metildopa, fentiazina e espirolactona) & * & - & - & - & - & - & + & - \\
\hline Bloqueadores dos canais de cálcio (diltiazem, nifedipina, verapramil) & * & - & - & - & - & - & + & - \\
\hline Outros estimulantes centrais contendo cafeína & * & - & - & - & + & - & - & - \\
\hline Drogas com ação estrogênica (rauwolfia) & * & - & - & - & - & - & + & - \\
\hline Drogas antiinflamatórias não esteroidais & * & - & - & - & - & - & + & - \\
\hline
\end{tabular}

*A especialidade apresenta, "como bula", um folheto indicativo com os demais produtos da empresa, não constando informações de possíveis interações.

+ Apresenta a informação.

- Não apresenta a informação.

ção em citar outras indicações de uso, relatadas em várias referências consultadas. No mais, observa-se a descrição de uma variedade de efeitos assinalados diferentemente em cada uma delas, mostrando que não há, por parte da autoridade reguladora, exigência de harmonização nas informações ao paciente por meio de bulas. Nas Tabelas 1, 2 e 3 é possível observar-se que, entre as oito amostras estudadas, não há homogeneidade nas informações ao paciente sobre indicações de uso, efeitos adversos e interações medicamentosas do P. ginseng. Ressalte-se que o parágrafo primeiro do art. 10 da $R D C n^{o} .140 / 0368$ indica que os fitoterápicos, assim como os medicamentos similares, deverão ter o conteúdo de suas bulas harmonizado conforme uma bula de referência.

Outra dificuldade adicional coloca-se neste âmbito, pois inúmeros autores assinalam o fato de que não se garante a homogeneidade da composição de diferentes preparações fitoterápicas. A Farmacopéia Americana 71, em sua 28a edição, apresenta especificações para a padronização de extrato e pó de P. ginseng, definindo a proporção mínima de ginsenosídeos combinados em de $3 \%$ $\mathrm{p} / \mathrm{p}$, além de estabelecer relação entre as quantidades de Rb2 e Rb1. 
Interações medicamentosas descritas na literatura científica comparadas às das embalagens e bulas de oito amostras de preparações industrializadas contendo Panax ginseng comercializadas na cidade de São Paulo, Brasil, 2004-2005.

\begin{tabular}{|c|c|c|c|c|c|c|c|c|}
\hline \multirow[t]{2}{*}{ Interações descritas em literatura } & \multicolumn{8}{|c|}{ Especialidades farmacêuticas analisadas } \\
\hline & 1 & 2 & 3 & 4 & 5 & 6 & 7 & 8 \\
\hline Com fenelzina (inibidor da MAO) $16,27,48,62,75$ & * & - & - & - & + & - & + & - \\
\hline Bebidas alcoólicas 24,62 & * & - & - & - & - & - & + & - \\
\hline Bloqueadores dos canais de cálcio (nifedipina) 16,27,48,62,75 & * & - & - & - & - & - & + & - \\
\hline Agentes antiplaquetários (varfarina) 16,25,37,45,62 & * & - & - & - & - & - & + & - \\
\hline Antiparasitários (benzimidazóis) 44 & * & - & - & - & - & - & - & - \\
\hline
\end{tabular}

* A especialidade apresenta, "como bula", um folheto indicativo com os demais produtos da empresa não constando informações de possíveis interações.

+ Apresenta a informação

- Não apresenta a informação.

Tabela 5

Posologia descrita em embalagem e bula de oito amostras de preparações industrializadas contendo Panax ginseng,

comercializadas na cidade de São Paulo, Brasil, 2004-2005.

\begin{tabular}{|c|c|c|c|c|}
\hline \multirow[t]{2}{*}{ Amostras } & \multicolumn{4}{|c|}{ Ingestão diária recomendada: 500 a $2.000 \mathrm{mg} /$ dia do pó seco de raízes 71} \\
\hline & Especialidade & Dosagem & Posologia & Quantidade diária indicada \\
\hline 1 & Ginseng (raiz/pó) & $400 \mathrm{mg}$ & 1 cápsula, 3 vezes ao dia & $\begin{array}{l}1.200 \mathrm{mg} \text { (correspondendo a } \\
18 \mathrm{mg} \text { em ginsenosídeo) }\end{array}$ \\
\hline 2 & Panax ginseng + associações & $300 \mathrm{mg}$ & 2 comprimidos ao dia & $\begin{array}{l}\text { 600mg (correspondendo a } \\
\text { 9mg em ginsenosídeo) }\end{array}$ \\
\hline 3 & Ginseng (raiz/pó) & $150 \mathrm{mg}$ & 1 ou 2 cápsulas, 3 vezes ao dia & $\begin{array}{l}\text { 900mg (correspondendo a } \\
\text { 13,5mg em ginsenosídeo) }\end{array}$ \\
\hline 4 & $\begin{array}{l}\text { Extrato padronizado de raízes com } \\
\qquad 4 \% \text { de ginsenosídeo }\end{array}$ & $100 \mathrm{mg}$ & 2 cápsulas ao dia & $\begin{array}{l}\text { 200mg (correspondendo a } \\
\text { 8mg em ginsenosídeo) }\end{array}$ \\
\hline 5 & Extrato seco a partir da raiz & $30 \mathrm{mg}$ & 2 a 4 cápsulas ao dia & $\begin{array}{l}\text { 120mg (correspondendo } \\
\text { a } 12 \mathrm{mg} \text { em ginsenosídeo) }\end{array}$ \\
\hline 6 & Extrato seco a partir da raiz + associações & $29,63 \mathrm{mg}$ & 2 cápsulas ao dia & $\begin{array}{l}\text { 59,26mg (correspondendo } \\
\text { a *mg de ginsenosídeo) }\end{array}$ \\
\hline 7 & $\begin{array}{c}\text { Extrato seco padronizado com 27-30mg } \\
\text { de ginsenosídeos totais }\end{array}$ & $100 \mathrm{mg}$ & 1 cápsula & $\begin{array}{l}\text { 100mg (correspondendo a } \\
\text { 30mg em ginsenosídeo) }\end{array}$ \\
\hline 8 & $\begin{array}{l}\text { Extrato padronizado em } 4 \% \text { de } \\
\text { ginsenosídeos + associações }\end{array}$ & $40 \mathrm{mg}$ & 2 cápsulas ao dia & $\begin{array}{l}\text { 80mg (correspondendo a } \\
3,2 \mathrm{mg} \text { em ginsenosídeo) }\end{array}$ \\
\hline
\end{tabular}

* Informação inexistente.

Aspecto de importância é abordado por Calixto 72 em sua análise no tocante a preparações com plantas medicinais; o autor considera a falta de padronização como responsável pela dificuldade em se avaliar corretamente a eficácia desse tipo de produto em ensaios clínicos, assim como a incidência de efeitos adversos. Harkey et al. 73 relataram, também, a falta de padronização na composição de várias amostras de preparações de $P$. ginseng, dentre outras comercializadas nos
Estados Unidos, demonstrando que essa falha ocorre em todos os lugares onde se comercializam esses produtos. Nesse contexto, Sengupta et al. ${ }^{74}$, com base na existência de princípios com ações farmacológicas opostas, assinalaram a ambigüidade dos efeitos do ginseng. Os dados sobre a posologia dos produtos avaliados, exibidos na Tabela 5, corroboram a observação destes autores. As quantidades diárias indicadas são todas diferentes, sem qualquer relação entre si, e 
consideradas apenas em relação à quantidade de extrato ou do pó da droga declaradas nas fórmulas. Podemos observar variação cerca de 13 vezes entre o maior e o menor valor. A avaliação em termos de concentração de ginsenosídeos é ainda mais prejudicada, pois nem todas as preparações apresentaram a correspondência entre estes e o extrato ou pó de ginseng presentes na formulação.

Verificou-se, ainda, nas bulas avaliadas, grandes quantidades de informações relativas à ação farmacológica em animais e não resultados de ensaios clínicos em humanos, estatisticamente significativos. Geralmente, são bulas longas, com conteúdos que poderiam ser suprimidos sem prejuízo da informação ao paciente e, portanto, mais concisas e objetivas.

As Tabelas 3 e 4 mostram que o item menos abordado nas bulas e/ou embalagens foi o da ocorrência de interações medicamentosas entre o ginseng e certos tipos de fármacos, o que poderá trazer conseqüências graves aos usuários, conforme dados da literatura científica. Nas raras ocasiões em que efeitos adversos são relatados, não estão diretamente relacionados ao produto, mas de forma vaga às espécies de ginseng estudadas. Em alguns casos, é recomendado ao usuário que, se algum efeito adverso ocorrer, ele deverá se utilizar de um medicamento específico para combatê-lo, sugerindo, assim, uma situação absurda, na qual o usuário toma um medicamento que só se faz necessário por ele consumir o ginseng.

Nenhuma das bulas avaliadas traz a recomendação para que o usuário informe ao seu médico o uso do ginseng, caso algum medicamento lhe seja prescrito concomitantemente, o que evitaria possíveis interações medicamentosas. Não constam, igualmente, observações para se evitar o produto no caso de restrição de uso, como, por exemplo, por idosos, para os quais interações medicamentosas são mais prováveis de ocorrer. As bulas para fitoterápicos devem apresentar informações que priorizem os alertas, visando à diminuição do risco associado à sua utilização, principalmente, pelos grupos de maior risco, como os idosos, crianças e particularmente em gestantes; neste caso, em virtude de efeitos de androgenização de recém-nascido de mãe usuária de ginseng, fato relatado na literatura.

Em face das novas exigências da legislação de bulas e rotulagem para medicamentos, é sugerido que, para os fitoterápicos, estas sejam mais específicas, levando-se em conta as particularidades desses produtos, com o intuito de se fornecer ao usuário o máximo de informações sobre as indicações de uso, reações adversas e interações medicamentosas decorrentes do seu consumo.

\section{Conclusão}

As únicas indicações aceitas como cientificamente comprovadas pela OMS no que se refere ao $P$. ginseng são as relacionadas às atividades profiláticas e restauradoras. Existem interações comprovadas entre o ginseng e alguns fármacos, que precisam ser informadas ao usuário destes produtos.

Ao contrário de grande parte dos fitoterápicos, as preparações provenientes de ginseng podem ser padronizadas quanto à composição, independentemente do método de obtenção do extrato, de acordo com as especificações preconizadas pela Farmacopéia Americana 71 .

Os idosos, as crianças e as gestantes precisam ser informados sobre as restrições de uso e do risco associado ao consumo de medicamentos que contenham ginseng, de modo a garantir a segurança de sua utilização. Por sua vez, a autoridade reguladora deve alertar o usuário em geral para o fato de que medicamentos que contenham essa substância poderão não ser inócuos, como estabelecido pela crença popular, e que, portanto, sua utilização deverá ser sempre revelada ao médico quando alguma medicação for indicada concomitantemente. 


\section{Resumo}

As informações contidas nas bulas e embalagem de medicamentos de diferentes fabricantes contendo $\mathrm{Pa}-$ nax ginseng foram analisadas comparativamente com os dados encontrados na literatura científica. Observaram-se bulas longas, com várias informações em relação às indicações de uso, muitas das quais sem comprovação em humanos, e todas foram deficitárias em relação aos efeitos adversos e interações medicamentosas. Foi observada falta de padronização quanto às posologias, em relação tanto ao extrato seco, quanto ao teor dos ginsenosídeos presentes. Constatou-se a falta de homogeneidade nas informações aos usuários sobre indicações de uso, efeitos adversos e interações medicamentosas entre as oito amostras estudadas.

Panax ginseng; Bulas de Medicamentos; Uso de Medicamentos

\section{Referências}

1. Washida D, Kitanaka S. Determination of polyacetylenes and ginsenosides in Panax species using high performance liquid chromatography. Chem Pharm Bull (Tokyo) 2003; 51:1314-7.

2. Park EK, Choo MK, Han MJ, Kim DH. Ginsenoside Rh1 possesses antiallergic and anti-inflammatory activities. Int Arch Allergy Immunol 2004; 133:11320.

3. Pennafort R. Brasileiro adere aos fitoterápicos. $\mathrm{O}$ Estado de São Paulo 2005; 4 abr.

4. Arias DC. Alternative medicines' popularity prompts concern: use of alternative and complementary remedies on the rise. http://www. medscape.com/viewarticle/484309 (acessado em 27/Set/2005).

5. Yu SM, Ghandour RM, Huang ZJ. Herbal supplement use among US women, 2000. J Am Med Womens Assoc 2004; 59:17-24.

6. D'Angelo L, Grimaldi R, Caravaggi M, Marcoli M, Perucca E, Lecchini S, et al. A double-blind, placebo-controlled clinical study on the effect of a standardized ginseng extract on psychomotor performance in healthy volunteers. J Ethnopharmacol 1986; 16:15-22.

7. Kennedy DO, Scholey AB. Ginseng: potential for the enhancement of cognitive performance and mood. Pharmacol Biochem Behav 2003; 75:687700.

8. Vogler RK, Pittler MH, Ernst E. The efficacy of ginseng: a systematic review of randomized clinical trials. Eur J Clin Pharmacol 1999; 55:567-75.

\section{Colaboradores}

Todas as autoras participaram do levantamento bibliográfico, leitura, discussão e seleção dos artigos; da composição do texto final, elaboração das tabelas, discussão e revisão das referências.
9. Choi HH, Jong HS, Park JH, Choi S, Lee JW, Kim TY, et al. Novel ginseng saponin metabolite induces apoptosis and down-regulates fibroblast growth factor receptor 3 in myeloma cells. Int J Oncol 2003; 23:1087-93.

10. Jeong CS, Hyun JE, Kim, YS. Ginsenoside RB1: the anti-ulcer constituent from the head of Panax ginseng. Arch Pharm Res 2003; 26:906-11.

11. Van Kampen J, Robertson H, Hagg T, Drobitch R. Neuroprotective actions of the ginseng extract G115 in two rodent models of Parkinson's disease. Exp Neurol 2003; 184:521-9.

12. Park EK, Choo MK, Han MJ, Kim DH. Antiallergic activity of ginsenoside Rh2. Biol Pharm Bull 2003, 26:1581-4.

13. Liou CJ, Li ML, Tseng J. Intraperitonial injection of ginseng extract enhances both immunoglobulin and cytokine production in mice. Am J Chin Med 2004; 32:75-88.

14. Joo SS, Won TJ, Lim MS, Lee D. Hematopoeitic effect of ginsenoside Rg3 in ICR mouse primary cultures and its application to a biological response modifier. Fitoterapia 2004; 75:337-41.

15. World Health Organization. Monographs on selected medicinal plants. Geneva: World Health Organization; 2001.

16. Singh VK, Agarwal SS, Gupta BM. Imunomodulatory effects of Panax ginseng extract. Planta Med 1984; 50:459-65. 
17. Kumar M, Sharma MK, Saxena PS, Kumar A. Radioprotective affect of Panax ginseng on the phosphatases and lipid peroxidation level in tests of Swiss albino mice. Biol Pharm Bull 2003; 26:308-12.

18. Yokozawa T, Satoh A, Cho EJ. Ginsenoside - Rd attenuates oxidative damage related to aging in senescence-accelerated mice. J Pharm Pharmacol 2004; 56:107-13.

19. Shen LH, Zhang JT. Ginsenoside Rg1 promotes proliferation of hippocampal progenitor cells. Neurol Res 2004; 26:422-8.

20. Kim SH, Park KS. Effects of Panax ginseng extract on lipid metabolism in humans. Pharmacol Res 2003; 48:511-3.

21. Kiefer D, Pantuso T. Panax ginseng. Am Fam Physician 2003; 68:1539-42.

22. Radad K, Gile K, Moldzio R, Saito H, Ishige K, Rausch WD. Ginsenosides Rb1 and Rgl effects on survival and neurite growth of MPP+ affected mesencephalic dopaminergic cells. J Neural Transm 2004; 111:37-45.

23. Mizumaki Y, Kurimoto M, Hirashima Y, Nishijima M, Kamiyama H, Nagai S, et al. Lipophilic fraction of Panax ginseng induces neuronal differentiation of PC12 cells and promotes neuronal survival of rat cortical neurons by protein kinase $\mathrm{C}$ dependent manner. Brain Res 2002; 950:254-60.

24. Kim HJ, Chun YJ, Park JD. Protection of rat liver microssomes against carbon tetrachloride-induced lipid peroxidation by red ginseng saponin through Cytochrome P450 inhibition. Planta Med 1997; 69:235-40.

25. Hikino H. Antihepatotoxic actions of ginsenosides from Panax ginseng roots. Planta Med 1985; 51:624.

26. Kwon YS, Jang KH, Jang IH. The effects of Korean ginseng on liver regeneration after parcial hepatectomy in dogs. J Vet Sci 2003; 4:83-92.

27. Kwon YS, Jang KH. The effect of korean red ginseng on liver regeneration after $70 \%$ hepatotectomy in rats. J Vet Med Sci 2004; 66:193-5.

28. Gross D, Shenkman Z, Bleiberg B, Dayan M, Gittelson M, Efrat R. Ginseng improves pulmonary functions and exercise capacity in patients with COPD. Monaldi Arch Chest Dis 2002; 57:242-6.

29. Choo MK, Park EK, Han MJ, Kim DH. Antiallergic activity of ginseng and its ginsenosides. Planta Med 2003; 69:518-22.

30. Seung-Hwan K, Kyung-Shin P. Effects of Panax ginseng extract on lipid metabolism in humans. Pharmacol Res 2003; 48:511-3.

31. Yoon M, Lee H, Jeong S, Kim JJ, Nicol CJ, Nam KW, et al. Peroxisome proliferator-activated receptor alpha is involved in the regulation of lipid metabolism by ginseng. Br J Pharmacol 2003; 138:295-302.

32. Kim DH, Moon YS, Jung JS, Min SK, Son BK, Suh HW, et al. Effects of ginseng saponin administered intraperitoneally on the hypothalamo-pituitaryadrenal axis in mice. Neurosci Lett 2003; 343:62-6.

33. Oshima Y, Khonno C, Hikino H. Isolation and hypoglycemic activity of panaxans I, J, K and L, glycans of Panax ginseng roots. J Ethnopharmacol $1985 ; 14: 255-9$.
34. Konno C, Sugiyama K, Kano M, Takahashi M, Hikino H. Isolation and hypoglycemic activity of panaxans A,B,C,D and E, glycans of Panax ginseng roots. Planta Med 1984; 50:434-6.

35. Konno C, Murakami M, Oshima Y, Hikino H. Isolation and hypoglycemic activity of panaxans Q, R, S, T and U: glycans of Panax ginseng roots. J Ethnopharmacol 1985; 14:69-74.

36. Tomoda M, Shimada K, Konno C, Sugiyama K, Hikino H. Partial structure of panaxan A, a hypoglycemic glycan of Panax ginseng roots. Planta Med 1984; 50:436-8.

37. Dey L, Xie JT, Wang A, Wu J, Maleckar SA, Yuan CS. Anti-hyperglycemic effects of ginseng: comparison between root and berry. Phytomedicine 2003; 10:600-5.

38. Sotaniemi EA, Haapakkoski E, Rautio A. Ginseng therapy in non-insulin dependent diabetic patients. Diabetes Care 1995; 18:1373-5.

39. Min KT, Koo BN, Kang JW, Bai SJ, Ko SR, Cho ZH. Effect of ginseng saponins on the recombinant serotonin type $3 \mathrm{~A}$ receptor expressed in xenopus oocytes: implication of possible application as an antiemetic. J Altern Complement Med 2003; 9:50510.

40. Hashimoto K, Satoh K, Murata P, Makino B, Sakakibara I, Kase Y, et al. Components of Panax ginseng that improve accelerated small intestinal transit. J Ethnopharmacol 2003; 84:115-9.

41. Chang YS, Seo EK, Gyllenhaal C, Block KI. Panax gineng: a role in cancer therapy? Integr Cancer Ther 2003; 2:13-33.

42. Helms S. Cancer prevention and therapeutics: Panax ginseng. Altern Med Rev 2004; 9:259-74.

43. Oh GS, Pae HO, Choi BM, Seo EA, Kim DH, Shin MK, et al. 20(S)-Protopanaxatriol, one of ginsenoside metabolites, inhibits inducible nitric oxide synthase and cyclooxygenase- 2 expressions through inactivation of nuclear factor-kappaB in RAW 264.7 macrophages stimulated with lipopolysaccharide. Cancer Lett 2004; 205:23-9.

44. Lapa AJ, Souccar C, Lima-Landman MTR, Godinho RO, Lima TCM. Farmacologia e toxicologia de produtos naturais. In: Simões CMO, Schenkel EP, Gosmann G, Mello JCP, Mentz LA, Petrovick PR, organizadores. Farmacognosia: da planta ao medicamento. 2a Ed. Florianópolis: EdUFSC; 2000. p. 181-96.

45. Coon JT, Ernst E. Panax ginseng: a systematic review of adverse effects and drug interactions. Drug Saf 2002; 25:323-44.

46. Punnonen R, Lukola A. Oestrogen-like effect of ginseng. Br Med J 1980; 281:1110.

47. Koren G. Maternal use of ginseng and neonatal androgenization [Letter]. JAMA 1990; 264:2866.

48. Awang DVS. Maternal use of ginseng and neonatal androgenization [Letter]. JAMA 1991; 265:1828.

49. Lee YJ, Jin YR, Lim WC, Park WK, Cho JY, Jang S, et al. Ginsenoside - Rb1 acts as a week phytoestrogen in MCF-7 human breast cancer cells. Arch Pharm Res 2003; 26:58-63.

50. Dega H, Laporte JL, Frances C, Herson S, Chosidow O. Ginseng as a cause for Stevens-Johnson syndrome? Lancet 1996; 347:1344. 
51. Vasquez I, Aguera-Ortiz LF. Herbal products and serious side effects: a case of ginseng-induced maniac episode. Acta Psychiatr Scand 2002; 105:76-7.

52. Tsung O, Cheng T. Panax ginseng is not a panacea. Arch Intern Med 2000; 27:3329.

53. Wiwanitkit V, Taungjaruwinai W. A case report of suspected ginseng allergy. MedGenMed 2004; 6:9.

54. Janetzky K, Morreale AP. Probable interactions between warfarin and ginseng. Am J Health Syst Pharm 1997; 54:692-3.

55. Kuo SC, Teng CM, Lee JG, Ko FN, Chen SC, Wu TS. Antiplatelet components in Panax ginseng. Planta Med 1990; 56:164-7.

56. Miller L. Herbal medicinals: selected clinical considerations focusing on known or potential drugherb interactions. Arch Intern Med 1998; 158:220011.

57. Dasgupta A. Review of abnormal laboratory: test results and toxic effects due to use of herbal medicines. Am J Clin Pathol 2003; 120:127-37.

58. Sparreboom A, Cox MC, Acharya MR, Figg WD. Herbal remedies in the United States: potencial adverse interactions with anticancer agents. J Clin Oncol 2004; 22:2489-503.

59. Yuang CS, Wej G, Dey L, Karrison T, Nahlik L, Maleckar S, et al. Brief communication: American ginseng reduces warfarin's effect in healthy patients, a randomized, controlled trial. Ann Intern Med 2004; 141:23-7.

60. Myers SP. Interactions between complementary medicines and warfarin. Australian Prescriber 2002; 25:54-6.

61. Jones BD, Runikis AM. Interactions of ginseng with phenelzine. J Clin Psychopharmacol 1987; 7:201-2.

62. Merino G, Molina AJ, Garcia JL, Oulido MM, Prieto JG, Alvarez AL. Ginseng increases intestinal elimination of albendazole sulfoxide in the rat. Comp Biochem Physiol C Toxicol Pharmacol 2003; 136:915.

63. Izzo A, Ernst E. Interactions between herbal medicines and prescribed drugs: a systematic review. Drugs 2001; 61:2163-75.

64. Brasil. Lei no. 6.360, de 23 de setembro de 1976 Dispõe sobre a vigilância sanitária a que ficam sujeitos os medicamentos, as drogas, os insumos farmacêuticos e correlatos, cosméticos, saneantes e outros produtos, e dá outras providências. Diário Oficial da União 1976; 24 set.
65. Brasil. Decreto no. 79.094, de 05 de janeiro de 1977. Regulamenta a Lei $n^{\circ}$. 6.360 de 23 de setembro de 1976, que submete a sistema de vigilância sanitária os medicamentos, as drogas, os insumos farmacêuticos e correlatos, cosméticos, produtos de higiene, saneantes e outros. Diário Oficial da União 1977; 8 jan.

66. Brasil. Resolução RDC nº. 92, de 23 de outubro de 2000. Dispõe sobre as normas para rotulagem de medicamentos e dá outras providências. Diário Oficial da União 2000; 24 out.

67. Brasil. Resolução RDC no. 47, de 28 de março de 2001. Dispõe sobre normas para embalagens de medicamentos. Diário Oficial da União 2001; 4 abr.

68. Brasil. Resolução RDC no. 140, de 29 de maio de 2003. Dispõe sobre as regras das bulas de medicamentos para pacientes e para profissionais de saúde. Diário Oficial da União 2003; 2 jun.

69. Brasil. Resolução RDC no. 333, de 19 de novembro de 2003. Dispõe sobre rotulagem de medicamentos e dá outras providências. Diário Oficial da União 2003; 21 nov.

70. Brasil. Resolução RDC no. 48, de 16 de março de 2004. Dispõe sobre o registro de medicamentos fitoterápicos. Diário Oficial da União 2004; 18 mar.

71. United States Pharmacopeial Convention. The United States pharmacopeia. Rockville: United States Phamacopeial Convention; 2004.

72. Calixto JB. Efficacy, safety, quality control, marketing and regulatory guidelines for herbal medicines (phytotherapeutic agents). Braz J Med Biol Res 2000; 33:179-89.

73. Harkey MR, Henderson GL, Gershwin ME, Stern JS, Hackman RM. Variability in commercial ginseng products of 25 preparations. Am J Clin Nutr 2001; 73:1101-6.

74. Sengupta S, Toh SA, Sellers LA, Skeppen JN, Koolwijk P, Leung HW, et al. The yin and yang in ginseng. Circulation 2004; 110:1219-25.

75. Price A, Gazewood J. Korean red ginseng effective for treatment of erectile dysfunction. J Fam Pract 2003; 52:20-1.

Recebido em 27/Dez/2005

Versão final reapresentada em 03/Out/2006

Aprovado em 16/Abr/2007 